\title{
Fuzzy Inventory Model without Shortage Using Trapezoidal Fuzzy Number with Sensitivity Analysis
}

\author{
D. Dutta ${ }^{1}$, Pavan Kumar ${ }^{2}$ \\ ${ }^{1,2}$ Department of Mathematics, National Institute of Technology, Warangal-506004, INDIA'
}

\begin{abstract}
In the present paper, an inventory model without shortages has been considered in a fuzzy environment. Our goal is to determine the optimal total cost and the optimal order quantity for the proposed inventory model. The Trapezoidal fuzzy numbers have been introduced in order to achieve this goal. The computation of economic order quantity $(E O Q)$ is carried out through defuzzification process by using signed distance method. The signed distance method is more applicable than the other methods of defuzzification. To illustrate the results of the proposed model, we have given two model examples and presented the computational results. Sensitivity for this model is also studied, which shows a linear relation between demand, EOQ, and total cost. The advantage of the proposed approach is that it is simple, gives a better result in relatively less computational work.
\end{abstract}

Keywords: Trapezoidal Fuzzy Numbers, Fuzzy Inventory Model, Defuzzification.

\section{Introduction}

Basically, an inventory consists of raw materials, work-in-progress, or finished goods. Effective inventory control is essential for manufacturing organizations for many reasons. One of them is the amount of money tied in inventory.

Thus, inventory control is very important field for both real world applications and research purpose. In conventional inventory models, the uncertainties are treated as randomness and are handled by using probability theory. The most widely used inventory model is the Economic Order Quantity (EOQ) Model, in which the successive operations are classified as supply and demand. The first quantitative treatment of inventory was the simple EOQ model. This model was developed by Harris[1]. Wilson[2] aroused interest in the EOQ model in academics and industries. Later, Hadley et al[3] analyzed many inventory systems.

In certain situations, uncertainties are due to fuzziness, primarily introduced by Zadeh[4], is applicable. In 1970, Zadeh et al[5] proposed some strategies for decision making in fuzzy environment. Jain[6] worked on decision making in the presence of fuzzy variables. Kacpryzk et al[7] discussed some long-term inventory policy-making through fuzzy-decision making models.

Wide applications of fuzzy set theory can be found in Zimmerman[8], and Park[10]. In basic EOQ model we identify the order size that minimizes the sum of annual costs of inventory holding and fixed setup to place orders. In this model, there are some assumptions:

- The demand is known, fixed and independent,

- Quantity discounts are not allowed

- Inventory replenishment is instantaneous

- Only variable costs are setup cost and inventory holding cost

- No safety stock.

Thus, EOQ model serves as a useful approximation to many real life problems. In literature, there are many papers on fuzzified problems of EOQ model. Urgeletti [9] treated EOQ model in fuzzy sense, and used triangular fuzzy number. Chen and Wang[11] used trapezoidal fuzzy number to fuzzify the order cost, inventory cost, and backorder cost in the total cost of inventory model without backorder. Then, they found the estimate of the total cost in the fuzzy sense by functional principle.

Vujosevic et al[12] used trapezoidal fuzzy number to fuzzify the order cost in the total cost of inventory model with backorder. Then, they got fuzzy total cost. They obtained the estimate of the total fuzzy cost through centroid to defuzzify.

Further, in a series of papers, Yao et al.[13,14,17], considered the fuzzified problems for the inventory with or without backorder models. In [13], they applied the extension principle to obtain the fuzzy total cost, and then, they defuzzified the fuzzy total cost by centroid. In [14], they considered the fuzzified problems for the inventory with or without backorder models using trapezoidal fuzzy number. In [17], they considered the fuzzified problems for the inventory without backorder models and they fuzzified the order quantity $q$ as the triangular fuzzy number. For defuzzification, the study shows that the signed distance method is better than centroid method Yao \& Lee [17]. 
Kao and Hsu[15] considered a single-period inventory model with fuzzy demand. Hsieh[16] analyzed some production inventory models in fuzzy sense and he proposed some optimal strategies. Syed \& Aziz [18] used trapezoidal fuzzy number. De and Rawat [19], proposed an EOQ model without shortage cost by using triangular fuzzy number. The total cost has been computed by using signed distance method.

We develop an inventory model considering carrying cost and set-up cost as trapezoidal fuzzy number, and the signed distance method is used for defuzzification. The average annual cost is expressed as a function of these two decision variables, and then determine the reorder point that minimizes the total cost. Usually, the model parameters exhibit variability, for example due to irregularities in fabrication or physical properties of a material. Thus the results obtained cannot be considered to be representative for the actual results. For this, we introduce Fuzzy set theoretic approach. Zadeh[4] defined the generalized mathematical operations for fuzzy numbers.

An algorithm is produced to find the optimal time-invariant strategy to find the replenishment to current inventory levels that maximizes the membership function for the decision. Finally we conclude that the associated result [19] has been changed to better accuracy, and sensitivity was also analyzed.

\section{Definitions and Preliminaries}

A fuzzy set $\tilde{A}$ on the given universal set $\mathrm{X}$ is a set of ordered pairs

$$
\tilde{A}=\left\{\left(\mathrm{x}, \mu_{A}(x): x \in X\right\}\right.
$$

where $\mu_{\tilde{A}}: X \rightarrow[0,1]$, is called membership function. The $\alpha$ - cut of $\tilde{A}$, is defined by $A_{\alpha}=\left\{\mathrm{x}: \mu_{A}(x)=\alpha, \alpha \geq\right.$ $0\}$. If $\mathrm{R}$ is the real line, then a fuzzy number is a fuzzy set $\tilde{A}$ with membership function $\mu_{\tilde{A}}: X \rightarrow[0,1]$, having the following properties:

(i) $\tilde{A}$ is normal, i.e., there exists $x \in R$ such that $\mu_{A}(x)=1$

(ii) $\tilde{A}$ is piece-wise continuous

(iii) $\operatorname{supp}(\tilde{A})=\operatorname{cl}\left\{x \in R: \mu_{A}(x)>0\right\}$, where cl represents the closure of a set

(iv) $\tilde{A}$ is a convex fuzzy set.

Definition 2.1: A trapezoidal fuzzy number $\tilde{A}=(\mathrm{a}, \mathrm{b}, \mathrm{c}, \mathrm{d})$ is represented with membership function $\mu_{\tilde{A}}$ as:

$$
\mu_{\tilde{A}}(x)=\left\{\begin{array}{cl}
L(x)=\frac{x-a}{b-a}, & \text { when } a \leq x \leq b ; \\
1 \quad & \text { when } b \leq x \leq c \\
R(x)=\frac{d-x}{d-c}, & \text { when } c \leq x \leq d \\
0 \quad, & \text { otherwise }
\end{array}\right.
$$

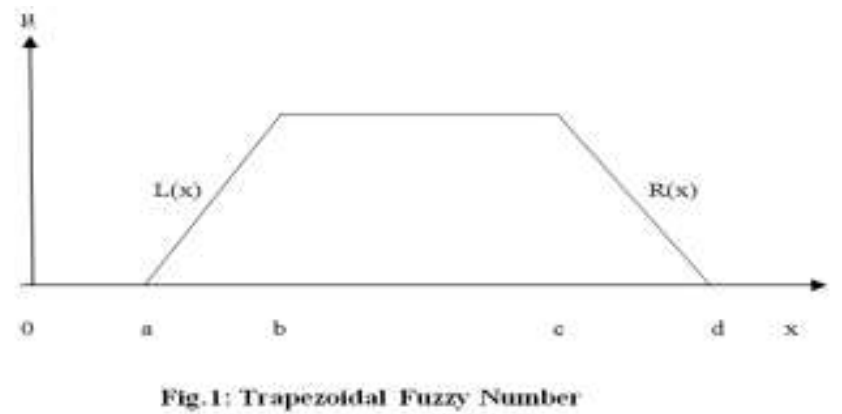

Definition 2.2: A fuzzy set is called in $L R$-Form, if there exist reference functions $L$ (for left), $R$ (for right), and scalars $\mathrm{m}>0$ and $\mathrm{n}>0$ with membership function

$$
\mu_{\tilde{A}}(x)=\left\{\begin{array}{lr}
L\left(\frac{\sigma-x}{m}\right), & \text { for } \mathrm{x} \leq \sigma ; \\
1, & \text { for } \sigma \leq \mathrm{x} \leq \gamma ; \\
R\left(\frac{x-\gamma}{n}\right), & \text { for } \mathrm{x} \geq \gamma .
\end{array}\right.
$$

Where $\sigma$ is a real number called the mean value of $\hat{A}, \mathrm{~m}$ and $\mathrm{n}$ are called the left and right spreads, respectively. The functions $L$ and $R$ map $R^{+} \rightarrow[0,1]$, and are decreasing. A $L R$-Type fuzzy number can be represented as $\tilde{A}=(\sigma, \gamma, m, n)_{L R}$.

Definition 2.3: Suppose $\tilde{A}=\left(a_{1}, a_{2}, a_{3}, a_{4}\right)$ and $\tilde{B}=\left(b_{1}, b_{2}, b_{3}, b_{4}\right)$ are two trapezoidal fuzzy numbers, then arithmetical operations are defined as: 
1. $\tilde{A} \oplus \tilde{B}=\left(a_{1}+b_{1}, a_{2}+b_{2}, a_{3}+b_{3}, a_{4}+b_{4}\right)$

2. $\tilde{A} \otimes \tilde{B}=\left(a_{1} b_{1}, a_{2} b_{2}, a_{3} b_{3}, a_{4} b_{4}\right)$

3. $\tilde{A} \ominus \tilde{B}=\left(a_{1}-b_{4}, a_{2}-b_{3}, a_{3}-b_{2}, a_{4}-b_{1}\right)$

4. $\tilde{A} \phi \tilde{B}=\left(\frac{a_{1}}{b_{4}}, \frac{a_{2}}{b_{3}}, \frac{a_{3}}{b_{2}}, \frac{a_{4}}{b_{1}}\right)$

5. $\alpha \otimes \tilde{A}= \begin{cases}\left(\alpha a_{1}, \alpha a_{2}, \alpha a_{3}, \alpha a_{4}\right), & \alpha \geq 0 \\ \left(\alpha a_{4}, \alpha a_{3}, \alpha a_{2}, \alpha a_{1}\right), & \alpha<0\end{cases}$

Definition 2.4: Let $\tilde{A}$ be a fuzzy set defined on $\mathrm{R}$. Then the signed distance of $\tilde{A}$ is defined as:

$$
\begin{aligned}
& \mathrm{d}(\tilde{A}, 0)=\frac{1}{2} \int_{0}^{1}\left[A_{L}(\alpha)+A_{R}(\alpha)\right] \mathrm{d} \alpha \\
& \text { where } \quad A_{\alpha}=\left[A_{L}(\alpha), A_{R}(\alpha)\right] \\
& =[a+(b-a) \alpha, d-(d-c) \alpha], \alpha \in[0,1],
\end{aligned}
$$

is $\alpha$ - cut of fuzzy set $\tilde{A}$, which is a close interval.

Remark: The signed distance $d(a, 0)=a$, for all $a, 0 \in R$. The meaning of Definition 2.4 is as the follows, if 0 $<a$ then the distance between $a$ and $O$ is $d(a, 0)=a$. If $a<0$ then the distance between $a$ and $O$ is $-d(a, 0)=-$ $a$. Therefore, we call $d(a, 0)=a$ is the signed distance between $a$ and 0 .

\subsection{Notations}

\section{Notations and Assumptions}

We define the following symbols:

c : holding cost per unit quantity per unit time

s $\quad$ : set up or ordering cost per order

$\mathrm{T} \quad$ : length of the plan

q : order quantity per cycle

$\mathrm{t}_{\mathrm{q}} \quad$ : length of a cycle

D : total demand over the planning time period $[0, \mathrm{~T}]$

TC : total cost for the period[0, T]

$\widetilde{\mathrm{TC}}$ : fuzzy total cost for the period $[0, \mathrm{~T}]$

$\mathrm{F}(\mathrm{q})$ : de-fuzzifyed total cost for $[0, \mathrm{~T}]$

$\mathrm{F}(\mathrm{q})^{*}$ : mimimum de-fuzzifyed total cost for $[0, \mathrm{~T}]$

$\mathrm{q}_{\mathrm{d}}^{*} \quad$ : optimal order quantity

\subsection{Assumptions}

In this paper, the following assumptions are considered:

(i) Total demand is considered as constant.

(ii) Time of plan is constant.

(iii) Shortages are not allowed.

(iv) Only holding cost and setup cost are fuzzy in nature.

\section{Proposed Inventory Model in Crisp Sense}

First, we deal an inventory model without shortages, in crisp environment. In this model, the economic lot size is obtained by the following model equation:

$$
\mathrm{q}=\sqrt{\frac{2 s D}{c T}} \text {, where } \frac{q}{t_{q}}=\frac{D}{T}
$$

The total cost for the period $[0, \mathrm{~T}]$ is equal to carrying cost plus ordering cost. That is:

$$
\mathrm{TC}=\frac{c T q}{2}+\frac{s D}{q}
$$

The optimum $q^{*}$ and $T C^{*}$ can be obtained by equating the first partial derivatives of TC to zero, and solving the resulting equations:

$$
\begin{aligned}
\text { Optimal order quantity } & q^{*}=\sqrt{\frac{2 s D}{c T}} \\
\text { Minimum total cost } \quad T C^{*} & =\sqrt{2 s c D T}
\end{aligned}
$$

Diagrammatic Representation: 


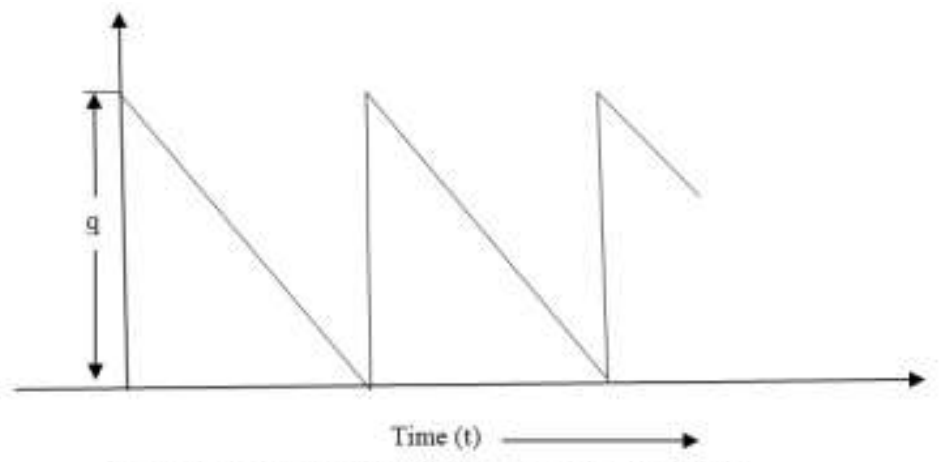

Fig.2: Variation of Quantity q with respect to time

\section{Proposed Inventory Model in Fuzzy Sense}

We consider the model in fuzzy environment. Since the ordering and holding cost are fuzzy in nature, we represent them by trapezoidal fuzzy numbers.

Let $\quad \tilde{c}$ : fuzzy carrying or holding cost per unit quantity per unit time

$\tilde{s}$ : fuzzy set up or ordering cost per order

The total demand and time of plan are considered as constants. Now we fuzzify total cost given in (6), the fuzzy total cost is given by:

$$
\widetilde{T C}=\frac{\tilde{C} \mathrm{Tq}}{2}+\frac{\tilde{s} \mathrm{D}}{\mathrm{q}}
$$

Our aim is to apply signed distance method to defuzzify the fuzzy total cost, and then obtain the optimal order quantity $\left(\mathrm{q}_{\mathrm{d}}^{*}\right)$ by using simple calculus technique.

Suppose $\tilde{c}=\left(c_{1}, c_{2}, c_{3}, c_{4}\right)$, and $\tilde{s}=\left(s_{1}, s_{2}, s_{3}, s_{4}\right)$ are fuzzy trapezoidal numbers, in LR form, where $0<\mathrm{s}<\mathrm{c}$ and $s_{1}, s_{2}, s_{3}, s_{4}, c_{1}, c_{2}, c_{3}$, and $c_{4}$ are known positive numbers.

From (9), we have:

$$
\begin{aligned}
\widetilde{T C}=\widetilde{T C}(\tilde{c}, \tilde{s}) & =\left[\tilde{c} \otimes \frac{T q}{2}\right] \oplus\left[\tilde{s} \otimes \frac{D}{q}\right] \\
& =\left[\left(\mathrm{c}_{1}, \mathrm{c}_{2}, \mathrm{c}_{3}, \mathrm{c}_{4}\right) \otimes\left(\frac{\mathrm{Tq}}{2}\right)\right] \oplus\left[\left(\mathrm{s}_{1}, \mathrm{~s}_{2}, \mathrm{~s}_{3}, \mathrm{~s}_{4}\right) \otimes\left(\frac{\mathrm{D}}{\mathrm{q}}\right)\right] \\
& =\left(\mathrm{c}_{1} \frac{\mathrm{Tq}}{2}, \mathrm{c}_{2} \frac{\mathrm{Tq}}{2}, \mathrm{c}_{3} \frac{\mathrm{Tq}}{2}, \mathrm{c}_{4} \frac{\mathrm{Tq}}{2}\right) \oplus\left(\mathrm{s}_{1} \frac{\mathrm{D}}{\mathrm{q}}, \mathrm{s}_{2} \frac{\mathrm{D}}{\mathrm{q}}, \mathrm{s}_{3} \frac{\mathrm{D}}{\mathrm{q}}, \mathrm{s}_{4} \frac{\mathrm{D}}{\mathrm{q}}\right) \\
& =\left(\mathrm{c}_{1} \frac{\mathrm{Tq}}{2}+\mathrm{s}_{1} \frac{\mathrm{D}}{\mathrm{q}}, \mathrm{c}_{2} \frac{\mathrm{Tq}}{2}+\mathrm{s}_{2} \frac{\mathrm{D}}{\mathrm{q}}, \mathrm{c}_{3} \frac{\mathrm{Tq}}{2}+\mathrm{s}_{3} \frac{\mathrm{D}}{\mathrm{q}}, \mathrm{c}_{4} \frac{\mathrm{Tq}}{2}+\mathrm{s}_{4} \frac{\mathrm{D}}{\mathrm{q}}\right) \\
& =(\mathrm{a}, \mathrm{b}, \mathrm{c}, \mathrm{d})(\mathrm{say})
\end{aligned}
$$

Now $A_{L}(\alpha)=a+(b-a) \alpha=c_{1} \frac{T q}{2}+s_{1} \frac{D}{q}+\left[\left(c_{2}-c_{1}\right) \frac{T q}{2}+\left(s_{2}-s_{1}\right) \frac{D}{q}\right] \alpha$

And $\quad A_{R}(\alpha)=d-(d-c) \alpha=c_{4} \frac{T q}{2}+s_{4} \frac{D}{q}-\left[\left(c_{4}-c_{3}\right) \frac{T q}{2}+\left(s_{4}-s_{3}\right) \frac{D}{q}\right] \alpha$

Defuzzifying $\widetilde{T C}$ in (10) by using signed distance method, we have:

$\mathrm{d}(\widetilde{T C}(\tilde{c}, \tilde{s}), 0)=\frac{1}{2} \int_{0}^{1}\left[A_{L}(\alpha)+A_{R}(\alpha)\right] \mathrm{d} \alpha$

$$
\begin{aligned}
& =\frac{1}{2}\left[\left(c_{1}+c_{4}\right) \frac{T q}{2}+\left(s_{1}+s_{4}\right) \frac{D}{q}\right]+\frac{1}{4}\left[\left(c_{2}+c_{3}-c_{1}-c_{4}\right) \frac{T q}{2}+\left(s_{2}+s_{3}-s_{1}-s_{4}\right) \frac{D}{q}\right] \\
& =\left[2\left(c_{1}+c_{4}\right)+\left(c_{2}+c_{3}-c_{1}-c_{4}\right)\right] \frac{T q}{8}+\left[2\left(s_{1}+s_{4}\right)+\left(s_{2}+s_{3}-s_{1}-s_{4}\right)\right] \frac{D}{4 q} \\
& =\left(c_{1}+c_{2}+c_{3}+c_{4}\right) \frac{T q}{8}+\left(s_{1}+s_{2}+s_{3}+s_{4}\right) \frac{D}{4 q} \\
& =F(q) \text { (say) }
\end{aligned}
$$

Computation of $\boldsymbol{q}_{d}^{*}$ at which $\mathrm{F}(\mathrm{q})$ is minimum:

$\mathrm{F}(\mathrm{q})$ is minimum when $\frac{\mathrm{dF}(\mathrm{q})}{\mathrm{dq}}=0$, and where $\frac{\mathrm{d}^{2} \mathrm{~F}(\mathrm{q})}{\mathrm{dq}^{2}}>0$.

Now, $\frac{d F(q)}{d q}=0$, gives the economic order quantity as:

$$
q_{d}^{*}=\sqrt{\frac{2 D}{T} \frac{\left(s_{1}+s_{2}+s_{3}+s_{4}\right)}{\left(c_{1}+c_{2}+c_{3}+c_{4}\right)}}
$$

Also, at $q=q_{d}^{*}$, we have $\frac{d^{2} F(q)}{d q^{2}}>0$

This shows that $\mathrm{F}(\mathrm{q})$ is minimum at $q=q_{d}^{*}$. And from(11), we find: 


$$
F(q)^{*}=\left(c_{1}+c_{2}+c_{3}+c_{4}\right) \frac{T q_{d}^{*}}{8}+\left(s_{1}+s_{2}+s_{3}+s_{4}\right) \frac{D}{4 q_{d}^{*}}
$$

Algorithm for Finding Fuzzy Total Cost and Fuzzy Optimal Order Quantity:

Step 1: Calculate total cost (TC) for the crisp model as given in eq.(5) for the given crisp values of $c, q, T$, and $D$.

Step 2: Now, determine fuzzy total cost $(\widetilde{T C})$ using fuzzy arithmetic operations on fuzzy carrying cost and fuzzy ordering cost, taken as fuzzy trapezoidal numbers.

Step 3: Use signed distance method for defuzzification of $\widetilde{T C}$. Then, find fuzzy optimal order quantity $\mathrm{q}_{\mathrm{d}}^{*}$, which can be obtained by putting the first derivative of $F(q)$ equal to zero and where second derivative of $F(q)$ is positive at $q=q_{d}^{*}$.

\section{Numerical Examples}

Example 1[19]. Crisp Model: Let $s=$ Rs. 20/- per unit, $c=$ Rs.12/- per unit, $D=500$ unit, $T=6$ days. Then economic order quantity and total cost are respectively, $q^{*}=16.67$ units, $T C=$ Rs. 1200 .

Fuzzy Model: $D=500, T=6$ days. Let $\tilde{S}=(15,19,21,24), \tilde{c}=(8,11,13,16)$. Then we find that $\mathrm{q}^{*}=16.56$ units, $\mathrm{F}(\mathrm{q})^{*}=$ Rs. $1192.47 /-$

Again, let $\tilde{s}=(15,18,22,24), \tilde{c}=(8,10,14,16)$. Then, we get the same value of $q^{*}=16.56$ units, and $\mathrm{F}(\mathrm{q})^{*}=$ Rs.1192.47/-

Table 1 : Sensitivity Analysis

\begin{tabular}{|c|c|c|c|c|c|}
\hline \multirow[t]{2}{*}{ S.No } & \multirow[t]{2}{*}{$\begin{array}{l}\text { Demand } \\
(D)\end{array}$} & \multicolumn{2}{|c|}{$\begin{aligned} \text { For } & \tilde{S}=(15,19,21,24), \\
& \tilde{c}=(8,11,13,16)\end{aligned}$} & \multicolumn{2}{|c|}{ 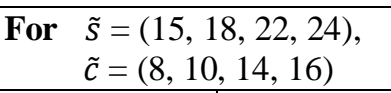 } \\
\hline & & $q_{d}^{*}$ & $\mathrm{~F}(\mathrm{q})^{*}$ & $q_{d}^{*}$ & $\mathrm{~F}(\mathrm{q})^{*}$ \\
\hline 1 & 450 & 15.71 & 1131.28 & 15.71 & 1131.28 \\
\hline 2 & 475 & 16.14 & 1162.24 & 16.14 & 1162.24 \\
\hline 3 & 500 & 16.56 & 1192.47 & 16.56 & 1192.47 \\
\hline 4 & 525 & 16.97 & 1221.92 & 16.97 & 1221.92 \\
\hline 5 & 550 & 17.37 & 1250.67 & 17.37 & 1250.67 \\
\hline
\end{tabular}

Example 2[19]. A company uses 24000 units of raw material which costs 1.25 rupees per unit. Placing each order costs rupees 2.5 and carrying cost $5.4 \%$ per year of the inventory. Find EOQ and the total inventory in both crisp and fuzzy sense.

Solution: Crisp Model

$s=22.5, c=1.25 * 5.4 \%=0.675, D=24000, T=1$.

We obtain $q^{*}=4000$ units, and $T C=$ Rs.853.82/-

Fuzzy Model

$s=22.5, \quad c=0.675, \quad D=24000, \quad T=1$.

Let $\tilde{s}=(15,20,25,30), \tilde{c}=(0.350,0.500,0.850,1.000)$. Then we find

$q^{*}=1341.64$ units, and $F(q)^{*}=$ Rs.804.98/-

Also, for $\tilde{s}=(15,19,26,30), \tilde{c}=(0.350,0.400,0.950,1.000)$, the values of $q^{*}$ and $F(q){ }^{*}$ are obtained.

Table 2: Sensitivity Analysis

\begin{tabular}{|l|l|l|l|l|l|}
\hline S.No & Demand & \multicolumn{2}{|l|}{$\begin{array}{l}\text { For } \\
\tilde{S}=(15,20,25,30),\end{array}$} & \multicolumn{2}{l|}{$\begin{array}{l}\text { For } \\
\tilde{s}=(15,19,26,30),\end{array}$} \\
& & \multicolumn{2}{l}{$\begin{array}{l}\tilde{c}=(0.350,0.500,0.850,1.000) \\
\tilde{c}=(0.350,0.400,0.950,1.00)\end{array}$} \\
\cline { 3 - 6 } & & $q_{d}^{*}$ & $F(q)^{*}$ & $q_{d}^{*}$ & $F(q)^{*}$ \\
\hline 1 & 23250 & 1320.51 & 792.30 & 1320.51 & 792.30 \\
2 & 23500 & 1327.59 & 796.55 & 1327.59 & 796.55 \\
3 & 23750 & 1334.63 & 800.78 & 1334.63 & 800.78 \\
$\mathbf{4}$ & $\mathbf{2 4 0 0 0}$ & $\mathbf{1 3 4 1 . 6 4}$ & $\mathbf{8 0 4 . 9 8}$ & $\mathbf{1 3 4 1 . 6 4}$ & $\mathbf{8 0 4 . 9 8}$ \\
5 & 24250 & 1348.61 & 809.16 & 1348.61 & 809.16 \\
6 & 24500 & 1355.54 & 813.32 & 1355.54 & 813.32 \\
7 & 24750 & 1362.44 & 817.46 & 1362.44 & 817.46 \\
8 & 25000 & 1369.30 & 821.58 & 1369.30 & 821.58 \\
\hline
\end{tabular}

\section{Observations :}


From Table-1, it is observed that:

(i) The Economic Order Quantity obtained by Signed Distance Method is closer to crisp Economic Order Quantity.

(ii) Total cost obtained by Signed distance Method is less than crisp total cost.

(iii) For different values of $\tilde{s}$ and $\tilde{c}$, changing only middle two spreads, the Economic Order Quantity remains fixed. The same is true for total cost.

From Table-2, it is observed that:

(i) Economic Order Quantity is more sensitive towards demand.

(ii) Total cost increases as the demand increases, and this increase is linear.

(iii) For different values of $\tilde{s}$ and $\tilde{c}$, changing only middle two spreads, the Economic Order Quantity remains fixed. The same is true for total cost.

\section{Conclusion}

In this paper, to defuzzify the set-up cost and carrying cost, the signed-distance method is used. The $E O Q$ has been calculated in both crisp and fuzzy environment. By using trapezoidal fuzzy number, defuzzification has been carried out and the corresponding changes have been observed. It is seen that $q_{d}^{*}$ increases/decreases with small amount which is under consideration and is within our level of expectation. Finally, we conclude that for an EOQ model if carrying cost and set up cost are expressed as fuzzy trapezoidal numbers, then the results obtained are much better \& economic than the case of triangular fuzzy numbers [19].

\section{References}

[1] Harris, F., Operations and cost, AW Shaw Co. Chicago, (1915).

[2] Wilson, R., A scientific routine for stock control. Harvard Business Review, 13, 1934, 116-128,

[3] Hadley, G., Whitin T.M., Analysis of inventory systems, Prentice-Hall, Englewood clipps, NJ, 1963.

[4] Zadeh L.A., Fuzzy sets, Information Control, 8, 338-353, 1965.

[5] Zadeh, L.A., Bellman, R.E., Decision Making in a Fuzzy Environment, Management Science, 17, 1970, 140-164.

[6] Jain, R., Decision making in the presence of fuzzy variables, IIIE Transactions on systems, Man and Cybernetics, 17, 1976, 698703.

[7] Kacpryzk, J., Staniewski, P., Long-term inventory policy-making through fuzzy-decision making models, Fuzzy Sets and Systems, $8,1982,117-132$.

[8] Zimmerman, H.J., Using fuzzy sets in operational Research, European Journal of Operational Research 13, 1983, 201-206.

[9] Urgeletti Tinarelli, G., Inventory control models and problems, European Journal of Operational Research, 14, $1983,1-12$.

[10] Park, K.S., Fuzzy Set Theoretical Interpretation of economic order quantity, IEEE Trans. Systems Man. Cybernet SMC, 17, 1987, 1082-1084.

[11] Chan,Wang, Backorder fuzzy inventory model under function principle, Information Science, 95, 1996, 1-2, 71-79.

[12] Vujosevic, M., Petrovic, D., Petrovic, R., EOQ Formula when Inventory Cost is Fuzzy, International Journal of Production Economics, 45, 1996, 499-504

[13] Yao, J.S., Lee, H.M., Fuzzy Inventory with or without backorder for fuzzy order quantity with trapezoidal fuzzy number, Fuzzy sets and systems, 105, 1999, 311-337.

[14] Yao, J.S., Lee, H.M., Economic order quantity in fuzzy sense for inventory without backorder model, Fuzzy Sets and Systems, 105, 1999, 13-31.

[15] Kao, C.K., Hsu, W.K., A single-period inventory model with fuzzy demand, Computers and Mathematics with Applications, 43, 2002, 841-848.

[16] Hsieh, C.H., Optimization of Fuzzy Production Inventory Models, Information Sciences, 146, 2002, 1-4, 29-40.

[17] Yao, J.S., Chiang, J., Inventory without back order with fuzzy total cost and fuzzy storing cost defuzzified by centroid and signed distance, European Journal of Operational Research, 148, 2003, 401-409.

[18] Syed, J.K., Aziz, L.A., Fuzzy inventory model without shortages using signed distance method, Applied Mathematics \& Information Sciences, 1(2), 2007, 203-209

[19] De, P.K., Rawat, A., A fuzzy inventory model without shortages using triangular fuzzy number, Fuzzy Information \& Engineering, $1,2011,59-68$ 